\title{
A Retrospective Study of Patients Presenting with Neck Masses to a Tertiary Care Center, Jeddah, Saudi Arabia
}

\author{
Majed Nasser Alnefaie ${ }^{1}$, Yousuf AlQurashi ${ }^{2}$, Asalh Tallat Saeedi ${ }^{1}$, Abdullah Ahmed Alamri ${ }^{1}$, Mohammed \\ Algethami ${ }^{1}$, Wejdan Baabdullah ${ }^{1}$, Adel Almaymuni ${ }^{1}$, Mazin Merdad ${ }^{2}$
}

${ }^{1}$ Faculty of Medicine, King Abdulaziz University, Jeddah, Saudi Arabia

${ }^{2}$ Department of Otolaryngology Head and Neck Surgery, Faculty of Medicine, King Abdulaziz University, Jeddah, Saudi Arabia

\section{Type of article: Original}

\begin{abstract}
Background: Studying the prevalence of different types of neck masses can enhance the appropriate and timely diagnosis of the etiology of a neck mass, which is critical in maintaining good prognosis. However, there is a paucity of studies in Saudi Arabia evaluating the prevalence of different types of neck masses.

Aim: This study was conducted to assess the etiologies of neck masses presenting to a large tertiary care hospital, and assess compliance with follow-up after diagnosis.

Methods: This retrospective study was carried out on all patients diagnosed with neck masses over the period from January 01, 2016 to April 30, 2018 at King Abdulaziz University Hospital, Jeddah, Saudi Arabia. Demographic and clinical data were collected from the medical records (age at diagnosis, type of neck mass, size of mass, and the procedure undertaken) and recorded on a structured form. Data analysis was performed by IBMSPSS Statistics version 21, using descriptive statistics, Chi-square test of independence, independent samples ttest and one-way ANOVA. All p-values $<0.05$ were considered statistically significant.

Results: A total of 195 patients were included in our study. The mean age was 44.6 years. The average size of neck masses was $4 \mathrm{~cm}$. A higher number of Non-Saudi patients received chemotherapy at our institution. Thyroid masses constituted $53.5 \%$ of the studied cases. Among patients diagnosed with lymphoma, $10.3 \%$ received chemotherapy treatment at our institution. The origin of the masses was significantly associated with gender $(\mathrm{p}<0.001$, Cramer's $\mathrm{V}=0.398)$, etiology $(\mathrm{p}=0.019$, Cramer's $\mathrm{V}=0.500)$ and malignancy $(\mathrm{p}<0.001$, Cramer's $\mathrm{V}=$ $0.466)$.

Conclusion: the majority of neck masses originated from thyroid or salivary glands. The rate of follow-up was low at our institution. The importance of follow-up cannot be over emphasized. Health education should be provided to all patients to raise their awareness about follow-up. Moreover, follow-up clinics should be provided, and written instructions as well as follow-up schedules should be handed to patients in need. We suggest having a dedicated endocrinology and oncology office and nurse to facilitate patient referral and follow-up.

Keywords: Neck; Mass; Thyroid; Lymphadenopathy; Neoplasm; Salivary glands
\end{abstract}

\section{Introduction}

The wide range of pathologies presented as neck masses include benign lesions to malignancies (1). Common causes for neck masses include lymphadenopathies (specific and non-specific, acute and chronic), metastatic carcinoma, lymphoma, thyroid masses (goiter, nodules, and cysts), and salivary gland swellings (sialadenitis, cysts, adenomas, and carcinomas) $(2,3)$. Congenital cystic lesions of the neck are uncommon (4). Out of these, thyroglossal duct cysts account for $70 \%$ of the congenital midline neck masses (5). As for the lateral aspect of the neck, branchial

\section{Corresponding author:}

Majed Nasser Alnefaie, Faculty of Medicine, King Abdulaziz University, Jeddah, Kingdom of Saudi Arabia. P.O. Box: 80215, Jeddah 21452. Tel: +966-12-6823614 Fax:+966-12-6823614, Email: Majed.n.md@gmail.com

Received: September 06, 2018, Accepted: December 28, 2018, Published: March 2019

iThenticate screening: December 25, 2018, English editing: February 05, 2019, Quality control: February 05, 2019

This article has been reviewed / commented by three experts

Ethics approval: Unit of Biomedical Ethics of King Abdulaziz University (Reg. ID: HA-02-J-008, Ref: 119-117)

(C) 2019 The Authors. This is an open access article under the terms of the Creative Commons Attribution-NonCommercialNoDerivs License, which permits use and distribution in any medium, provided the original work is properly cited, the use is non-commercial and no modifications or adaptations are made. 
anomalies are estimated as the most predominant congenital masses. Congenital anomalies of the lateral neck include dermoids and cystic hygromas (lymphangiomas), which constitute about $5 \%$ of all benign tumors of infancy and childhood (6). Infection is another important cause of neck masses; both bacterial (most commonly Staphylococcus and Streptococcus species) and viral infections can be the etiology of the abscesses (7). Tuberculous lymphadenitis is a common etiology in young adults, with a female preponderance in developing countries (8). However, it is a rare cause in developed countries (9). The most common cause of pediatric neck masses is inflammatory; other etiologies may include congenital or neoplastic lesions (10). Head and neck tumors account for $5 \%$ of all childhood cancers (11). The common pediatric head and neck tumors are lymphomas (59\%), rhabdomyosarcomas $(13 \%)$, thyroid tumors $(10 \%)$, nasopharyngeal carcinomas $(5 \%)$, neuroblastomas $(5 \%)$, nonrhabdomyosarcoma soft-tissue sarcomas $(4.5 \%)$, salivary gland malignancies $(2.5 \%)$, and malignant teratomas $(1 \%)$ (12). It is known that a malignant neoplasm in the neck can arise as a primary tumor or as a metastasis from the upper aero digestive tract or a distant site (1). Head and neck cancers (HNC) are reported to be the ninth most common cancer worldwide. It is a prevalent malignancy in Saudi Arabia, mostly reported in the southwestern region of the country (13). Radiation exposure to the head and neck is a risk factor for cancer of the salivary glands (14).

Neck masses can cause a lot of concern and complications to patients. Head and neck malignant tumors are associated with poor survival rate (13). Even if the mass is benign, complications can arise such as infection or problems with breathing or swallowing. Studying the prevalence of different types of neck masses can enable the treating physician to formulate an effective differential diagnosis, reach a diagnosis, and start management promptly. Despite the high prevalence of HNC and their poor survival rate (13), there is a lack of studies in Saudi Arabia about the prevalence of different types of neck masses. Therefore, this study was carried out with the general objective of assessing the etiologies of neck masses presenting to a large tertiary care hospital in Jeddah, Saudi Arabia. The specific objectives were: 1) to determine the prevalence of different types of neck masses and 2) to determine the rate of follow-up after discharge from the hospital.

\section{Material and Methods}

\subsection{Study design and settings}

A retrospective study was conducted from January 1, 2016 to April 30, 2018 at King Abdulaziz University Hospital (KAUH), a teaching, tertiary care Hospital located in Jeddah, Saudi Arabia.

\subsection{Eligibility criteria}

The inclusion criterion was inpatients and outpatients - of all age groups -who presented with neck masses at KAUH during the period from January 1, 2015 to April 30, 2018. Patients with incomplete data were excluded from the study.

\subsection{Study methods}

The electronic medical records of patients with neck masses were reviewed to identify patients who fulfilled the eligibility criteria. Demographic and clinical data were obtained from the medical records, including the patients' age at diagnosis, type of neck mass, size of mass, and the procedure undertaken. The study variables were recorded in a structured form that was designed for data collection.

\subsection{Statistical analysis}

Data analysis was performed using the IBMC-SPSS $\odot$ Statistics version 21 (IBMC Corp., Armonk, NY, USA). Numerical variables were expressed as mean \pm standard deviation, whereas categorical variables were summarized as frequency and percentage. Comparison of numerical variables between groups was achieved using Student $\mathrm{T}$ test (for two groups) and one-way ANOVA (for three groups, followed by Tukey test as a post-hoc test). Association between two categorical variables was tested using Pearson's Chi square test of independence and Cramer's V Coefficient as a measure of association. All $\mathrm{p}$-values $<0.05$ were considered statistically significant.

\subsection{Ethics of research on electronic records of patients}

Ethical Approval was obtained from the Unit of Biomedical Ethics of King Abdulaziz University (Registration ID: HA-02-J-008, Ref: 119-117) prior to the commencement of the study. To preserve the confidentiality of the patients' information, the researchers' access to the patients' records was limited to the studied variables. In addition, patients were assigned code numbers (known only to the researchers) so that the anonymity of the patients and their personal information were not violated. 


\section{Results}

Searching through the patients' records retrieved 195 patients with neck masses who satisfied the eligibility criteria. All participants underwent open surgical intervention as a primary treatment in the period from January 1, 2015 to April 30,2018 . The mean age of the population studied was $44.6 \pm 17.7$ years old; pediatric patients constituted only $7.2 \%$ of all cases. Female patients outnumbered males $(64.1 \%$ and $35.9 \%$ respectively). Saudi participants constituted $59.5 \%$, while $40.5 \%$ were Non Saudi (Table 1).

Table 1. Sociodemographic data of the studied patients $(\mathrm{n}=195)$.

\begin{tabular}{|l|l|l|l|}
\hline Sociodemographic data & \multicolumn{2}{l|}{$\mathrm{n}=195$} \\
\hline Age (years) & Min - Max & $4.0-94.0$ \\
\cline { 2 - 4 } & Mean \pm SD & $44.6 \pm 17.7$ \\
\hline \multirow{2}{*}{ Age group } & Pediatric & 14 & $7.2 \%$ \\
\cline { 2 - 4 } & Adult & 181 & $92.8 \%$ \\
\hline \multirow{2}{*}{ Gender } & Female & 125 & $64.1 \%$ \\
\cline { 2 - 4 } & Male & 70 & $35.9 \%$ \\
\hline \multirow{2}{*}{ Nationality } & Non-Saudi & 79 & $40.5 \%$ \\
\cline { 2 - 4 } & Saudi & 116 & $59.5 \%$ \\
\hline
\end{tabular}

Min: minimum; Max: Maximum; SD: standard deviation

Table 2. Neck mass characteristics and management ( $\mathrm{n}=195)$.

\begin{tabular}{|c|c|c|c|c|}
\hline \multicolumn{3}{|c|}{ Characteristics and management of mass } & \multirow{2}{*}{\multicolumn{2}{|c|}{$\begin{array}{l}n=195 \\
0.4-23.0\end{array}$}} \\
\hline \multirow[t]{20}{*}{ Characteristics of neck masses } & \multirow[t]{2}{*}{ Mass size $(\mathrm{cm})$} & Min - Max & & \\
\hline & & Mean \pm SD & \multicolumn{2}{|c|}{$4.1 \pm 2.8$} \\
\hline & \multirow[t]{3}{*}{ Aetiology of the mass } & Congenital & 7 & $3.6 \%$ \\
\hline & & Inflammatory & 12 & $6.2 \%$ \\
\hline & & Neoplastic & 176 & $90.3 \%$ \\
\hline & \multirow[t]{2}{*}{ Neoplastic } & Benign & 102 & $52.3 \%$ \\
\hline & & Malignant & 93 & $47.7 \%$ \\
\hline & \multirow[t]{13}{*}{ Origin } & Thyroid & 101 & $51.8 \%$ \\
\hline & & Salivary glands & 27 & $13.8 \%$ \\
\hline & & Lymph node & 26 & $13.3 \%$ \\
\hline & & Larynx & 12 & $6.2 \%$ \\
\hline & & Tongue & 7 & $3.6 \%$ \\
\hline & & Soft tissues & 6 & $3.1 \%$ \\
\hline & & Pharynx & 5 & $2.6 \%$ \\
\hline & & Fetal remnants & 3 & $1.5 \%$ \\
\hline & & Tonsil & 2 & $1.0 \%$ \\
\hline & & Maxillofacial & 2 & $1.0 \%$ \\
\hline & & Parathyroid & 2 & $1.0 \%$ \\
\hline & & Oral & 1 & $0.5 \%$ \\
\hline & & Palate & 1 & $0.5 \%$ \\
\hline \multirow[t]{13}{*}{ Management of neck masses } & \multirow[t]{9}{*}{ Procedure } & Diagnostic & 19 & $11.3 \%$ \\
\hline & & Biopsy & 19 & $9.7 \%$ \\
\hline & & Therapeutic & 149 & $88.7 \%$ \\
\hline & & Neck dissection & 19 & $9.7 \%$ \\
\hline & & Partial thyroidectomy & 19 & $9.7 \%$ \\
\hline & & Salivary gland excision & 26 & $13.3 \%$ \\
\hline & & Soft tissue excision & 4 & $2.1 \%$ \\
\hline & & Total thyroidectomy & 81 & $41.5 \%$ \\
\hline & & Other & 27 & $13.8 \%$ \\
\hline & \multirow[t]{2}{*}{ Chemotherapy in KAUH } & No & 175 & $89.7 \%$ \\
\hline & & Yes & 20 & $10.3 \%$ \\
\hline & \multirow[t]{2}{*}{ Follow up with endocrinologist } & No & 123 & $63.1 \%$ \\
\hline & & Yes & 72 & $36.9 \%$ \\
\hline
\end{tabular}


The mean size of the masses was $4.1 \pm 2.8 \mathrm{~cm}$. The majority of masses were neoplastic (90.3\%), while congenital and inflammatory masses accounted for 3.6\% and 6.2\% respectively. Malignant masses represented $47.7 \%$ of all cases. About half the masses originated from the thyroid gland, other most frequently encountered masses originated from salivary glands (13.8\%), lymph nodes (13.3\%), larynx $(6.2 \%)$, tongue $(3.6 \%)$, soft tissues of the neck $(3.1 \%)$ and the pharynx (2.6\%). Biopsy was performed in $11.3 \%$ of cases. Therapeutic procedures included total thyroidectomy in $41.5 \%$ of cases, while partial thyroidectomy was performed in $9.7 \%$. Other common procedures performed included excision of salivary glands (13.3\%), neck dissection (9.7\%) and soft tissue excision (2.1\%). Only 36.9\% of patients followed up at our institution after their surgery. Chemotherapy was received by $10.3 \%$ of patients (Table 2 ).

Table 3. Histopathologic findings of the studied neck masses $(n=195)$.

\begin{tabular}{|c|c|c|c|c|c|}
\hline \multicolumn{4}{|l|}{ Findings } & $\mathrm{n}$ & $\%$ \\
\hline \multirow{6}{*}{\multicolumn{2}{|c|}{ Congenital }} & \multirow[t]{2}{*}{ Lymph Node $(n=2)$} & Lymphangioma & 1 & $0.5 \%$ \\
\hline & & & Teratoma & 1 & $0.5 \%$ \\
\hline & & Salivary Glands $(\mathrm{n}=1)$ & Hemangioma & 1 & $0.5 \%$ \\
\hline & & \multirow[t]{2}{*}{ Fetal Remnants $(n=3)$} & Brachial Cleft Cyst & 1 & $0.5 \%$ \\
\hline & & & Thyroglossal Cyst & 2 & $1.0 \%$ \\
\hline & & Tongue $(n=1)$ & Hemangioma & 1 & $0.5 \%$ \\
\hline \multirow{6}{*}{\multicolumn{2}{|c|}{ Inflammatory }} & \multirow[t]{2}{*}{ Lymph Node $(n=4)$} & Granuloma & 1 & $0.5 \%$ \\
\hline & & & Reactive Hyperplasia & 3 & $1.5 \%$ \\
\hline & & Pharynx $(n=1)$ & Granuloma & 1 & $0.5 \%$ \\
\hline & & \multirow[t]{2}{*}{ Salivary Glands $(\mathrm{n}=2)$} & Inflammatory Cyst & 1 & $0.5 \%$ \\
\hline & & & Reactive Hyperplasia & 1 & $0.5 \%$ \\
\hline & & Thyroid $(n=5)$ & Thyroiditis & 5 & $2.6 \%$ \\
\hline \multirow[t]{31}{*}{ Neoplastic } & \multirow[t]{12}{*}{ Benign } & \multirow[t]{3}{*}{ Larynx $(n=3)$} & Papilloma & 1 & $0.5 \%$ \\
\hline & & & Glandular And Stromal Hyperplasia & 1 & $0.5 \%$ \\
\hline & & & Squamous Hyperplasia & 1 & $0.5 \%$ \\
\hline & & \multirow[t]{2}{*}{ Parathyroid $(n=2)$} & Parathyroid Adenoma & 1 & $0.5 \%$ \\
\hline & & & Parathyroid Hyperplasia & 1 & $0.5 \%$ \\
\hline & & \multirow[t]{3}{*}{ Salivary Glands $(n=19)$} & Adenoma & 15 & $7.7 \%$ \\
\hline & & & Lipoma & 2 & $1.0 \%$ \\
\hline & & & Warthin's Tumor & 2 & $1.0 \%$ \\
\hline & & Soft Tissues $(n=3)$ & Lipoma & 3 & $1.5 \%$ \\
\hline & & \multirow[t]{3}{*}{ Thyroid $(\mathrm{n}=57)$} & Multinodular Goiter & 40 & $20.5 \%$ \\
\hline & & & Solitary Nodule & 3 & $1.5 \%$ \\
\hline & & & Thyroid Adenoma & 14 & $7.1 \%$ \\
\hline & \multirow[t]{19}{*}{ Malignant } & Larynx $(n=9)$ & Squamous Cell Carcinoma & 9 & $4.6 \%$ \\
\hline & & \multirow[t]{2}{*}{ Lymph Node $(n=20)$} & Metastatic Carcinoma & 8 & $4.0 \%$ \\
\hline & & & Lymphoma & 12 & $6.2 \%$ \\
\hline & & \multirow[t]{2}{*}{ Maxillofacial $(n=2)$} & Adenocarcinoma & 1 & $0.5 \%$ \\
\hline & & & Ameloblastoma & 1 & $0.5 \%$ \\
\hline & & Oral/palate/tongue $(\mathrm{n}=8)$ & Squamous Cell Carcinoma & 8 & $4.1 \%$ \\
\hline & & \multirow[t]{3}{*}{ Pharynx $(n=4)$} & Metastastic Carcinoma & 1 & $0.5 \%$ \\
\hline & & & Nasopharyngeal Carcinoma & 2 & $1.0 \%$ \\
\hline & & & Squamous Cell Carcinoma & 1 & $0.5 \%$ \\
\hline & & \multirow[t]{4}{*}{ Salivary Glands $(\mathrm{n}=5)$} & Acinic Cell Carcinoma & 1 & $0.5 \%$ \\
\hline & & & Adenocarcinoma & 2 & $1.0 \%$ \\
\hline & & & Lipomosarcoma & 1 & $0.5 \%$ \\
\hline & & & Warthin's Tumor & 1 & $0.5 \%$ \\
\hline & & \multirow[t]{2}{*}{ Soft Tissues $(n=3)$} & Basal Cell Carcinoma & 2 & $1.0 \%$ \\
\hline & & & Squamous Cell Carcinoma & 1 & $0.5 \%$ \\
\hline & & \multirow[t]{3}{*}{ Thyroid $(n=39)$} & Lymphoma & 2 & $1.0 \%$ \\
\hline & & & Multinodular Goiter & 4 & $2.1 \%$ \\
\hline & & & Thyroid Papillary Carcinoma & 33 & $16.9 \%$ \\
\hline & & Tonsil $(n=2)$ & Squamous Cell Carcinoma & 2 & $1.0 \%$ \\
\hline
\end{tabular}


Histopathologic findings of the studied neck masses showed that the most common benign thyroid masses were multinodular goiter $(20.5 \%$ of all cases), followed by thyroid adenoma $(7.1 \%)$, while the most common malignant thyroid mass was papillary carcinoma $(16.9 \%)$. The most common benign salivary gland masses were adenomas (7.7\%) and the commonest malignant were adenocarcinoma (1\%). Non-malignant masses of lymph nodes were mainly inflammatory and showed reactive follicular hyperplasia (1.5\%). Malignant neoplasms in lymph nodes comprised mainly of lymphoma (6.2\%), followed by metastatic carcinoma (4\%). Malignant laryngeal masses were squamous cell carcinoma (4.6\%) (Table 3 ).

The results of Pearson's Chi square test of independence showed that there was no significant association between age groups and each of gender $(p=0.553$, Cramer's $V=0.042)$ and origin of the masses $(p=0.603$, Cramer's $\mathrm{V}=0.251)$. The Chi square test showed also that there were significant associations between age and nationality $(\mathrm{p}=0.038$, Cramer's V $=0.149$ ) as well as age and the etiology of masses ( $\mathrm{p}=0.011$, Cramer's Cramer's $\mathrm{V}=0.268)$. Independent-samples t-test showed that mass size did not differ significantly between the two age groups $(\mathrm{p}=0.653$ ) (Table 4).

Table 4. Association between age groups and each of sociodemographic data and mass characteristics $(\mathrm{n}=195)$.

\begin{tabular}{|c|c|c|c|c|c|c|c|}
\hline \multicolumn{2}{|c|}{ Sociodemographic characteristics } & \multicolumn{2}{|c|}{ Pediatric $(n=14)$} & \multicolumn{2}{|c|}{ Adult $(n=181)$} & \multirow{3}{*}{$\begin{array}{l}\text { Test statistics } \\
X^{2}=0.352 \\
V=0.042\end{array}$} & \multirow{3}{*}{$\frac{\mathrm{p} \text {-value }}{0.553}$} \\
\hline \multirow[t]{2}{*}{ Gender } & Female & 10 & $71.4 \%$ & 115 & $63.5 \%$ & & \\
\hline & Male & 4 & $28.6 \%$ & 66 & $36.5 \%$ & & \\
\hline \multirow[t]{2}{*}{ Nationality } & Non-Saudi & 2 & $14.3 \%$ & 77 & $42.5 \%$ & \multirow{2}{*}{$\begin{array}{l}X^{2}=4.305 \\
V=0.149\end{array}$} & \multirow[t]{2}{*}{$0.038^{*}$} \\
\hline & Saudi & 12 & $85.7 \%$ & 104 & $57.5 \%$ & & \\
\hline \multirow[t]{2}{*}{ Mass size $(\mathrm{cm})$} & Min - Max & 1.40 & 12.00 & 0.40 & 23.00 & \multirow[t]{2}{*}{$\mathrm{t}=0.450$} & \multirow[t]{2}{*}{0.653} \\
\hline & Mean \pm SD & 4.46 & 3.04 & 4.11 & 2.79 & & \\
\hline \multirow[t]{3}{*}{ Aetiology } & Congenital & 3 & $21.4 \%$ & 4 & $2.2 \%$ & \multirow{3}{*}{$\begin{array}{l}X^{2}=8.958 \\
V=0.268\end{array}$} & \multirow[t]{3}{*}{$0.011^{*}$} \\
\hline & Inflammatory & 1 & $7.1 \%$ & 12 & $6.6 \%$ & & \\
\hline & Neoplastic & 10 & $71.4 \%$ & 165 & $91.2 \%$ & & \\
\hline \multirow[t]{7}{*}{ Origin } & Larynx & 0 & $0.0 \%$ & 12 & $6.6 \%$ & \multirow{7}{*}{$\begin{array}{l}X^{2}=13.003 \\
V=0.251\end{array}$} & \multirow[t]{7}{*}{0.603} \\
\hline & Lymph node & 3 & $21.4 \%$ & 23 & $12.7 \%$ & & \\
\hline & Salivary glands & 2 & $14.3 \%$ & 25 & $13.8 \%$ & & \\
\hline & Soft tissues & 0 & $0.0 \%$ & 6 & $3.3 \%$ & & \\
\hline & Thyroid & 6 & $42.9 \%$ & 95 & $52.5 \%$ & & \\
\hline & Tongue & 1 & $7.1 \%$ & 6 & $3.3 \%$ & & \\
\hline & Others & 2 & $14.3 \%$ & 14 & $7.7 \%$ & & \\
\hline
\end{tabular}

Min: minimum; Max: Maximum; SD: standard deviation; V: Cramer's V; * significant at $\mathrm{p}<0.05$.

The association between gender and other sociodemographic data and mass characteristics was determined using Chi square test of independence. There were no significant associations between gender of patients and each of age $(\mathrm{p}=0.553$, Cramer's $\mathrm{V}=0.042)$, nationality $(\mathrm{p}=0.158$, Cramer's $\mathrm{V}=0.101)$ and etiology of masses $(\mathrm{p}=0.174$, Cramer's $\mathrm{V}=0.143$ ). A significant association was found between gender and the origin of masses ( $p<0.001$, Cramer's $\mathrm{V}=0.398)$ as higher percentage of female patients had masses that originated from thyroid $(62.4 \% \mathrm{vs.} 32.9 \%$ in males), while higher percentage of males had masses originating from the larynx (15.7\% vs. $0.8 \%$ in women) and lymph nodes (18.6\% vs. $10.4 \%$ in women). Independent-samples t-test showed that mass size did not differ significantly between men and women $(\mathrm{p}=0.246)$ (Table 5$)$.

As regards the etiology of the neck masses, analysis using the Chi square test of independence revealed a significant association between etiology of neck masses and the age group ( $\mathrm{p}=0.011$, Cramer's $\mathrm{V}=0.268$ ), as a higher percentage of congenital masses presented in the pediatric age group $(42.9 \% \mathrm{vs} .7 .7 \%$ of inflammatory and $5.7 \%$ of neoplastic etiology). The etiology of masses was significantly associated also with the origin ( $p=0.019$, Cramer's $\mathrm{V}=0.500$ ) as all masses originating from the larynx were neoplastic. There were no significant associations between the etiology of the mass and gender $(\mathrm{p}=0.174$, Cramer's $\mathrm{V}=0.143)$ and nationality $(\mathrm{p}=0.223$, Cramer's $\mathrm{V}=0.136)$. One-way ANOVA test (followed by post-hoc Tukey test) revealed that the mean size of the masses differed significantly between the etiology groups, as the mean size was significantly smaller in congenital masses compared to neoplasms ( $\mathrm{p}=0.007)$ (Table 6). Moreover, the results of Chi square test of independence showed the presence of significant association between the nature of the masses (benign or malignant) and their origin $(p<0.001$, Cramer's $\mathrm{V}=0.466$ ), as a higher percentage of thyroid and salivary gland lesions were benign, while the majority of lymph 
http://www.ephysician.ir

node, laryngeal, tongue masses were malignant. There were no significant associations between the malignancy of the masses and gender $(p=0.137$, Cramer's $V=0.107)$ and nationality $(p=0.167$, Cramer's $V=0.099)$. Independent samples $\mathrm{T}$ test showed that the mean age of patients was significantly higher in malignant masses than the benign ( 47.5 vs. 42 years; $p=0.030$ ); it showed also the absence of significant difference in mass size between the two groups ( $\mathrm{p}=0.495)$ (Table 7 and Figure 1).

Table 5. Association between gender and each of sociodemographic data and mass characteristics $(\mathrm{n}=195)$.

\begin{tabular}{|c|c|c|c|c|c|c|c|}
\hline \multirow{2}{*}{\multicolumn{2}{|c|}{ Variables }} & \multicolumn{4}{|c|}{ Gender } & \multirow[t]{2}{*}{ Test statistic } & \multirow[t]{2}{*}{ p-value } \\
\hline & & \multicolumn{2}{|c|}{ Female $(n=125)$} & \multicolumn{2}{|c|}{ Male $(n=70)$} & & \\
\hline \multirow[t]{2}{*}{ Age } & Min - Max & 10.0 & 94.0 & 4.0 & 79.0 & \multirow[t]{2}{*}{$\mathrm{t}=0.391$} & \multirow[t]{2}{*}{0.697} \\
\hline & Mean \pm SD & 44.2 & 17.8 & 45.3 & 17.6 & & \\
\hline \multirow[t]{2}{*}{ Age group } & Pediatric & 10 & $8.0 \%$ & 4 & $5.7 \%$ & \multirow{2}{*}{$\begin{array}{l}X^{2}=0.352 \\
V=0.042\end{array}$} & \multirow[t]{2}{*}{0.553} \\
\hline & Adult & 115 & $92.0 \%$ & 66 & $94.3 \%$ & & \\
\hline \multirow[t]{2}{*}{ Nationality } & Non-Saudi & 46 & $36.8 \%$ & 33 & $47.1 \%$ & \multirow{2}{*}{$\begin{array}{l}X^{2}=1.992 \\
V=0.101\end{array}$} & \multirow[t]{2}{*}{0.158} \\
\hline & Saudi & 79 & $63.2 \%$ & 37 & $52.9 \%$ & & \\
\hline \multirow[t]{2}{*}{ Mass size $(\mathrm{cm})$} & Min - Max & 0.80 & 12.00 & 0.40 & 23.00 & \multirow[t]{2}{*}{$\mathrm{t}=1.168$} & \multirow[t]{2}{*}{0.246} \\
\hline & Mean \pm SD & 3.93 & 2.15 & 4.49 & 3.68 & & \\
\hline \multirow[t]{3}{*}{ Aetiology } & Congenital & 2 & $1.6 \%$ & 5 & $7.1 \%$ & \multirow{3}{*}{$\begin{array}{l}X^{2}=3.771 \\
V=0.143\end{array}$} & \multirow[t]{3}{*}{0.174} \\
\hline & Inflammatory & 9 & $7.2 \%$ & 4 & $5.7 \%$ & & \\
\hline & Neoplastic & 114 & $91.2 \%$ & 61 & $87.1 \%$ & & \\
\hline \multirow[t]{7}{*}{ Origin } & Larynx & 1 & $0.8 \%$ & 11 & $15.7 \%$ & \multirow{7}{*}{$\begin{array}{l}\mathrm{X}^{2}=30.906 \\
\mathrm{~V}=0.398\end{array}$} & \multirow[t]{7}{*}{$<0.001^{*}$} \\
\hline & Lymph node & 13 & $10.4 \%$ & 13 & $18.6 \%$ & & \\
\hline & Salivary glands & 15 & $12.0 \%$ & 12 & $17.1 \%$ & & \\
\hline & Soft tissues & 4 & $3.2 \%$ & 2 & $2.9 \%$ & & \\
\hline & Thyroid & 78 & $62.4 \%$ & 23 & $32.9 \%$ & & \\
\hline & Tongue & 3 & $2.4 \%$ & 4 & $5.7 \%$ & & \\
\hline & Others & 11 & $8.8 \%$ & 5 & $7.1 \%$ & & \\
\hline
\end{tabular}

Min: minimum; Max: Maximum; SD: standard deviation; V: Cramer's V; * significant at $\mathrm{p}<0.05$.

Table 6. Association between etiology of masses and each of sociodemographic data and mass characteristics ( $\mathrm{n}=195)$.

\begin{tabular}{|c|c|c|c|c|c|c|c|c|c|}
\hline \multirow{2}{*}{\multicolumn{2}{|c|}{ Variables }} & \multicolumn{6}{|c|}{ Aetiology of neck masses } & \multirow{4}{*}{$\begin{array}{l}\text { Test } \\
\text { statistic } \\
\mathrm{F}=5.231\end{array}$} & \multirow[t]{2}{*}{ p-value } \\
\hline & & \multicolumn{2}{|c|}{ Congenital $(n=7)$} & \multicolumn{2}{|c|}{ Inflammatory $(\mathrm{n}=12)$} & \multicolumn{2}{|c|}{ Neoplastic $(n=176)$} & & \\
\hline \multirow[t]{2}{*}{ Age } & Min - Max & 4.0 & 54.0 & 13.0 & 80.0 & 12.0 & 94.0 & & $0.007^{*}$ \\
\hline & Mean $\pm \mathrm{SD}$ & $24.9^{\mathrm{a}}$ & 16.8 & 41.2 & 18.5 & $45.7^{\mathrm{a}}$ & 17.2 & & \\
\hline \multirow[t]{2}{*}{ Age group } & Pediatric & 3 & $42.9 \%$ & 1 & $7.7 \%$ & 10 & $5.7 \%$ & \multirow{2}{*}{$\begin{array}{l}X^{2}=13.987 \\
V=0.268\end{array}$} & \multirow[t]{2}{*}{$0.011^{*}$} \\
\hline & Adult & 4 & $57.1 \%$ & 12 & $92.3 \%$ & 165 & $94.3 \%$ & & \\
\hline \multirow[t]{2}{*}{ Gender } & Female & 2 & $28.6 \%$ & 9 & $69.2 \%$ & 114 & $65.1 \%$ & \multirow{2}{*}{$\begin{array}{l}X^{2}=3.771 \\
V=0.143\end{array}$} & \multirow[t]{2}{*}{0.174} \\
\hline & Male & 5 & $71.4 \%$ & 4 & $30.8 \%$ & 61 & $34.9 \%$ & & \\
\hline \multirow[t]{2}{*}{ Nationality } & Non-Saudi & 1 & $14.3 \%$ & 7 & $53.8 \%$ & 71 & $40.6 \%$ & \multirow{2}{*}{$\begin{array}{l}X^{2}=3.383 \\
V=0.136\end{array}$} & \multirow[t]{2}{*}{0.223} \\
\hline & Saudi & 6 & $85.7 \%$ & 6 & $46.2 \%$ & 104 & $59.4 \%$ & & \\
\hline \multirow{2}{*}{$\begin{array}{l}\text { Mass size } \\
(\mathrm{cm})\end{array}$} & Min - Max & 1.80 & 8.80 & 1.00 & 10.00 & .40 & 23.00 & \multirow[t]{2}{*}{$\mathrm{F}=0.144$} & \multirow[t]{2}{*}{0.877} \\
\hline & Mean \pm SD & 3.60 & 2.47 & 4.11 & 2.84 & 4.16 & 2.82 & & \\
\hline \multirow[t]{7}{*}{ Origin } & Larynx & 0 & $0.0 \%$ & 0 & $0.0 \%$ & 12 & $6.9 \%$ & \multirow{7}{*}{$\begin{array}{l}X^{2}=46.473 \\
V=0.500\end{array}$} & \multirow[t]{7}{*}{$0.019 *$} \\
\hline & Lymph node & 2 & $28.6 \%$ & 4 & $30.8 \%$ & 20 & $11.4 \%$ & & \\
\hline & Salivary glands & 1 & $14.3 \%$ & 2 & $15.4 \%$ & 24 & $13.7 \%$ & & \\
\hline & Soft tissues & 0 & $0.0 \%$ & 0 & $0.0 \%$ & 6 & $3.4 \%$ & & \\
\hline & Thyroid & 0 & $0.0 \%$ & 6 & $46.2 \%$ & 95 & $54.3 \%$ & & \\
\hline & Tongue & 1 & $14.3 \%$ & 0 & $0.0 \%$ & 6 & $3.4 \%$ & & \\
\hline & Others & 3 & $42.9 \%$ & 1 & $7.7 \%$ & 12 & $6.9 \%$ & & \\
\hline
\end{tabular}

Min: minimum; Max: Maximum; SD: standard deviation;

a: significant difference between congenital and neoplastic; V: Cramer's V; * significant at $\mathrm{p}<0.05$. 
Table 7. Association between the nature of neck masses (malignancy) and each of sociodemographic data and mass characteristics $(n=195)$.

\begin{tabular}{|c|c|c|c|c|c|c|c|}
\hline \multirow{2}{*}{\multicolumn{2}{|c|}{ Variables }} & \multicolumn{4}{|c|}{ Histopathology } & \multirow{4}{*}{$\begin{array}{l}\text { Test } \\
\text { statistic } \\
\mathrm{t}=2.190\end{array}$} & \multirow{4}{*}{$\begin{array}{l}\mathrm{p} \text {-value } \\
0.030^{*}\end{array}$} \\
\hline & & \multicolumn{2}{|c|}{ Benign $(n=103)$} & \multicolumn{2}{|c|}{ Malignant $(\mathrm{n}=92)$} & & \\
\hline \multirow[t]{2}{*}{ Age } & Min - Max & 4.0 & 85.0 & 14.0 & 94.0 & & \\
\hline & Mean \pm SD & 42.0 & 16.6 & 47.5 & 18.4 & & \\
\hline \multirow[t]{2}{*}{ Age group } & Pediatric & 9 & $8.7 \%$ & 5 & $5.4 \%$ & \multirow{2}{*}{$\begin{array}{l}X^{2}=796 \\
V=0.064\end{array}$} & \multirow[t]{2}{*}{0.372} \\
\hline & Adult & 94 & $91.3 \%$ & 87 & $94.6 \%$ & & \\
\hline \multirow[t]{2}{*}{ Gender } & Female & 71 & $68.9 \%$ & 54 & $58.7 \%$ & \multirow{2}{*}{$\begin{array}{l}X^{2}=2.213 \\
V=0.107\end{array}$} & \multirow[t]{2}{*}{0.137} \\
\hline & Male & 32 & $31.1 \%$ & 38 & $41.3 \%$ & & \\
\hline \multirow[t]{2}{*}{ Nationality } & Non-Saudi & 37 & $35.9 \%$ & 42 & $45.7 \%$ & \multirow{2}{*}{$\begin{array}{l}\mathrm{X}^{2}=1.909 \\
\mathrm{~V}=0.099\end{array}$} & \multirow[t]{2}{*}{0.167} \\
\hline & Saudi & 66 & $64.1 \%$ & 50 & $54.3 \%$ & & \\
\hline \multirow[t]{2}{*}{ Mass size $(\mathrm{cm})$} & Min - Max & 0.5 & 12.0 & 0.4 & 23.0 & \multirow[t]{2}{*}{$\mathrm{t}=0.684$} & \multirow[t]{2}{*}{0.495} \\
\hline & Mean \pm SD & 4.3 & 2.5 & 4.0 & 3.1 & & \\
\hline \multirow[t]{7}{*}{ Origin } & Larynx & 3 & $2.9 \%$ & 9 & $9.8 \%$ & \multirow{7}{*}{$\begin{array}{l}X^{2}=40.802 \\
V=0.466\end{array}$} & \multirow[t]{7}{*}{$<0.001^{*}$} \\
\hline & Lymph node & 6 & $5.8 \%$ & 20 & $21.7 \%$ & & \\
\hline & Salivary glands & 22 & $21.4 \%$ & 5 & $5.4 \%$ & & \\
\hline & Soft tissues & 3 & $2.9 \%$ & 3 & $3.3 \%$ & & \\
\hline & Thyroid & 62 & $60.2 \%$ & 39 & $42.4 \%$ & & \\
\hline & Tongue & 1 & $1.0 \%$ & 6 & $6.5 \%$ & & \\
\hline & Others & 6 & $5.8 \%$ & 10 & $10.9 \%$ & & \\
\hline
\end{tabular}

Min: minimum; Max: Maximum; SD: standard deviation; V: Cramer's V; * significant at $\mathrm{p}<0.05$.

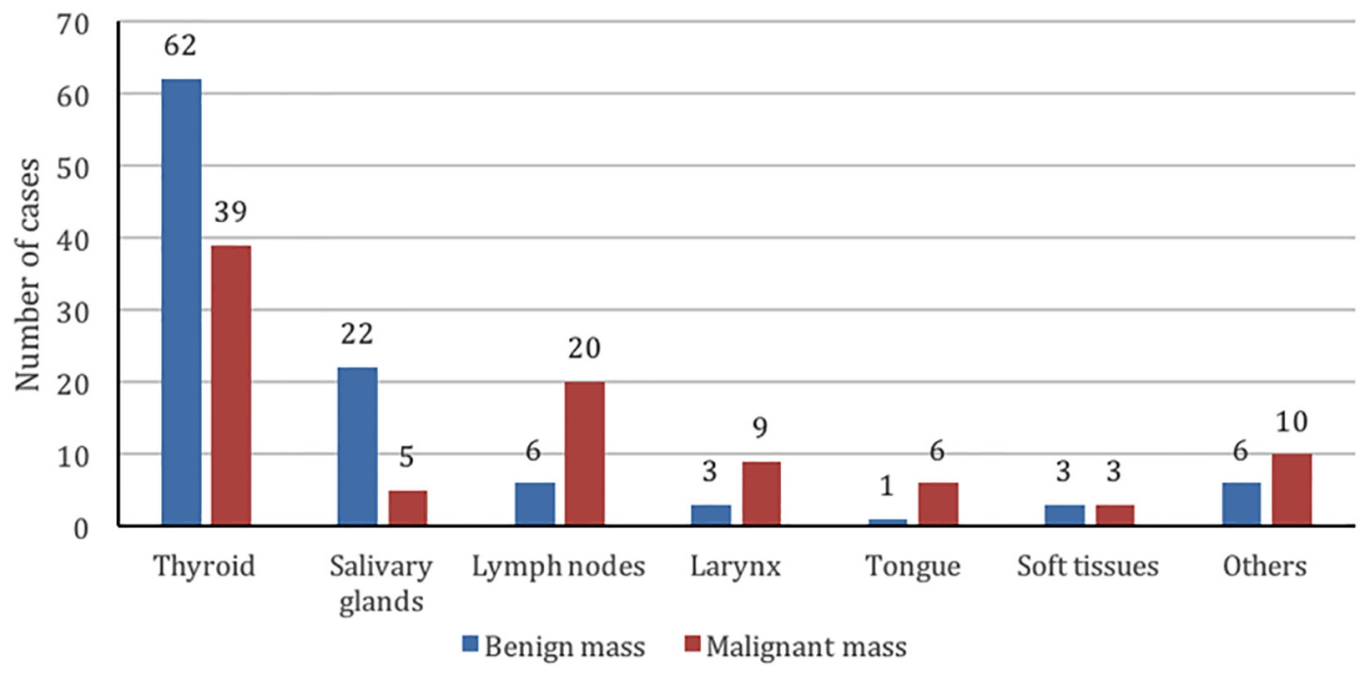

Figure 1. Incidence of common benign and malignant neoplastic masses $(\mathrm{n}=195)$.

\section{Discussion}

This study was carried out to assess the etiologies of neck masses presenting to a large tertiary care hospital. We found that the majority of neck masses were neoplastic, followed in prevalence by inflammatory then congenital masses. As regards the origin of the studied neck masses, about half the masses aroused from the thyroid gland. Our findings are in line with the results of previously published studies $(1,15)$. A lower prevalence of thyroid masses (35.7\%) was reported by Irani et al. (1) in Tehran. Other common origins for masses in our study included salivary glands and lymph nodes. Compared to our results, Irani et al. (1) reported a higher prevalence of lymph node masses (34.6\%) and studies by Irani et al. (1) and Rathod and Parmar (15) reported a slightly lower rate of salivary gland masses (about 11\%). This high incidence of thyroid masses could be attributed to the fact that thyroid disorders are relatively common among the Saudi population, with 7\% of the population having hypothyroidism (16). Also, thyroid cancer is the second most common cancer among Saudi women (17). Other common sites for neck masses included salivary glands, lymph nodes and larynx. 
In the present study, slightly less than half the masses were malignant, an incidence which is similar to that in the study by Naeimi et al. (18). Most thyroid and salivary gland masses were benign, while most lymph node, laryngeal and tongue masses were malignant. These findings agree with previous studies $(1,19,20)$. Dean and Hay (19) and Al-Salama et al (20) reported most thyroid masses to be benign, while malignant thyroid lesions accounted only for $5-10 \%$ of thyroid nodules. Shishegar et al. (21) and Irani et al. (1) found that benign masses of the salivary glands were more common than malignant neoplasms. Irani et al. (1) reported also that malignant neoplasms of lymph nodes, particularly lymphoma, were more common than benign lymphadenopathy. On the other hand, the percentage of malignant thyroid lesions in our study is higher than that reported by previous studies in Saudi Arabia (22). This may be partially attributed to the nature of our institution, being a referral tertiary care facility. Lymphoma was reported as the most common malignant neck mass in Iran, while thyroid cancer followed $(18,23)$.

The most common benign thyroid masses were multinodular goiter, followed by thyroid adenoma, while the most common malignant thyroid mass was papillary carcinoma. This is in accordance with Bouq et al. (22), Refeidi et al. (24), Irani et al. (1) and Al-Amri (25) who found papillary carcinoma to be the most common thyroid cancer. In salivary glands, the commonest benign masses were adenomas and the commonest malignant were adenocarcinoma. Non-malignant masses of lymph nodes were mainly inflammatory and showed reactive follicular hyperplasia. Malignant neoplasms in lymph nodes comprised mainly of lymphoma, followed by metastatic carcinoma. Malignant laryngeal masses were squamous cell carcinoma. Among all risk factors for the development of squamous cell carcinoma, cigarette smoking and excessive consumption of alcohol are the most important, and they have a synergistic effect (26). The rate of smoking in Saudi Arabia is high and increasing for both cigarettes and shisha use (27). In KSA $64.6 \%$ of patients with head and neck squamous cell carcinoma were found to be males, with $41.5 \%$ of patients being smokers and $26.2 \%$ have been users of chewable tobacco, pepper and oil $(27,28)$.

Pediatric cases accounted only for $7.2 \%$ of all patients in our study. As regards the association of neck masses with age groups, a significantly higher percentage of congenital masses was found in the pediatric group when compared with adults. However, neoplastic masses were present in the majority of both age groups, which contradicts the results of Irani et al. (1) who found that developmental cystic lesions were the most common masses in the pediatric group, and neoplastic lesions were the third most common masses. Moreover, other studies have reported that the most common type of neck masses in children is inflammatory mass, particularly lymphadenitis (29). Most pediatric masses were benign, which was stated also by Dickson and Davidoff (30) and Irani et al. (1). All laryngeal masses were detected in adults, which is anticipated in light of the risk factors of laryngeal cancers (smoking, alcoholism, human papilloma virus), which are not present in pediatric age group.

As regards gender differences observed in this study, the majority of laryngeal masses occurred in men, while a significantly higher percentage of women had thyroid masses. This is in line with previous studies that reported a predilection of the female sex for thyroid masses $(1,8,22,31,32)$ and of the male sex for laryngeal cancer $(30,33)$. Markou et al. (33) reported a male to female ratio of 30:1 in laryngeal cancers. The predominance of laryngeal cancers in men may be due to higher cigarette consumption than females, as smoking was reported to be significantly associated with laryngeal cancer $(33,34)$. Neoplastic masses in men tended to be malignant, contrary to the female, but this difference did not reach statistical significance. This increase in percentage of malignant masses in men may be explained by the fact that most laryngeal and lymph node masses in this study occurred in men, and most of them were malignant.

In the current study, a significantly higher frequency of Non-Saudis received chemotherapy at our institution than Saudis $(40 \%$ and $20 \%$ respectively). Saudis may have sought treatment at other local or international oncology specialized centers. However, verification is needed to ascertain this assumption or to detect non-compliance with chemotherapy. Saudi and Non-Saudi thyroid patients had nearly the same low frequency of follow-up with endocrinologists at KAUH (38.8\% and 34.2\%). One explanation for the general low rate of follow-up at our outpatient clinics is related to overbooking and crowdedness.

\section{Study strengths and limitations}

The present study had many points of strength, being the first study to evaluate the prevalence of different types of neck masses in this region of Saudi Arabia. Also, our study included both male and female patients from all age groups. However, our study was subject to some limitations; further analysis of the association between different 
pathologies and sex, as well as age groups, was needed. Moreover, the sample size was relatively small as the study was carried out in a single center.

\section{Conclusions}

In conclusion, the majority of neck masses in our series originated from thyroid or salivary glands. The rate of follow-up was low at our institution. The importance of follow-up cannot be over emphasized. Health education should be provided to all patients to raise their awareness about follow-up. Moreover, follow-up clinics should be provided, and written instructions as well as follow-up schedules should be handed to patients in need. We suggest having a dedicated endocrinology and oncology office and nurse to facilitate patient referral and follow-up. Future research - preferably a multi-centered study - is needed to determine the prevalence of various types of neck masses in different age and sex groups.

\section{Acknowledgments:}

The authors express their gratitude and great appreciation to Ms. Amna Alkhaldi who assisted with data collection and retrieving the patients' records; and would like to thank all the staff members of the department, for their cooperation and assistance.

\section{Conflict of Interest:}

There is no conflict of interest to be declared.

Authors' contributions:

All authors contributed to this project and article equally. All authors read and approved the final manuscript.

\section{References:}

1) Irani S, Zerehpoush FB, Sabeti S. Prevalence of Pathological Entities in Neck Masses: A Study of 1208 Consecutive Cases. Avicenna Journal of Dental Research. 2016; 8. Available from: http://avicennajdr.portal.tools/?page $=$ article\&article $i d=25614$.

2) Andrén-Sandberg A, Brugger LE, Lumley JSP. Hamilton Bailey's physical signs. Demonstrations of physical signs in clinical surgery. Oxford, UK: Butterworth Heinemann; 1997.

3) Emerick K, Lin D. Differential Diagnosis of a neck mass.UpToDate. 2016.

4) Koeller KK, Alamo L, Adair CF, Smirniotopoulos JG. Congenital cystic masses of the neck: radiologicpathologic correlation. Radiographics. 1999; 19(1): 121-46. doi: 10.1148/radiographics.19.1.g99ja06121. PMID: 9925396.

5) Allard RHB. The thyroglossal cyst. Head Neck Surg. 1982; 5: 134-46. doi: 10.1002/hed.2890050209.

6) Hernandez M, Chowdhury R, Woods J, Cabrera J, Hardigan PC. Management of suppurative cervical lymphadenitis in a healthy 24-year-old man. J Am Osteopath Assoc. 2011; 111: 49-51. PMID: 21258017.

7) Cengiz B, Kara A, Kanra G, Seçmeer G, Ceyhan M, Ozen M. Acute neck infections in children. Turk J Pediatr. 2004; 46: 153-8. PMID: 15214745.

8) Dean DS, Gharib H. Epidemiology of thyroid nodules. Best Pract Res Clin Endocrinol Metab. 2008; 22: 901-11. doi: 10.1016/j.beem.2008.09.019. PMID: 19041821.

9) Yates MD, Grange JM. Bacteriological survey of tuberculous lymphadenitis in southeast England, 19811989. J Epidemiol Community Health. 1992; 46: 332-5. PMID: 1431701, PMCID: PMC1059594.

10) Meier JD, Grimmer JF. Evaluation and management of neck masses in children. American family physician. 2014; 89.

11) Sengupta S, Pal R. Clinicopathological correlates of pediatric head and neck cancer. J Cancer Res Ther. 2009; 5(3): 181-5. doi: 10.4103/0973-1482.57123. PMID: 19841559.

12) Mittra $P$, Bharti R, Pandey MK. Role of fine needle aspiration cytology in head and neck lesions of paediatric age group. J Clin Diagn Res. 2013; 7(6): 1055-8. doi: 10.7860/JCDR/2013/5384.3067. PMID: 23905102, PMCID: PMC3708197.

13) Alhazzazi TY, Alghamdi FT. Head and Neck Cancer in Saudi Arabia: a Systematic Review. Asian Pac J Cancer Prev. 2016; 17(8): 4043-8. PMID: 27644659.

14) Mayne ST, Morse DE, Winn DM. Cancers of the Oral Cavity and Pharynx. Cancer Epidemiology and Prevention Oxford University Press. 2009.

15) Rathod GB, Parmar P. Fine needle aspiration cytology of swellings of head and neck region. Indian J Med Sci. 2012; 66(3-4): 49-54. doi: 10.4103/0019-5359.110896. PMID: 23603620. 
16) Al-Geffari M, Ahmad NA, Al-Sharqawi AH, Youssef AM, AlNaqeb D, Al-Rubeaan K. Risk factors for thyroid dysfunction among type 2 diabetic patients in a highly diabetes mellitus prevalent society. International journal of endocrinology. 2013; 2013.

17) Saudi Cancer Registry. Cancer Incidence Report 2014. Riyadh, Saudi Arabia: Saudi Health Council; 2014.

18) Naeimi M, Sharifi A, Erfanian Y, Velayati A, Izadian S, Golparvar S. Differential diagnosis of cervical malignant lymphadenopathy among Iranian patients. Saudi Med J. 2009; 30: 377-81. PMID: 19271066.

19) Dean DS, Hay ID. Prognostic indicators in differentiated thyroid carcinoma. Cancer control. 2000; 7: 22939. doi: 10.1177/107327480000700302. PMID: 10832109.

20) Al-Salamah SM, Kamran Khalid F, Bismar HA, CAES C. nodular goiter. Saudi Med J 2002;23:947-952.

21) Shishegar M, Ashraf MJ, Azarpira N, Khademi B, Hashemi B, Ashrafi A. Salivary gland tumors in maxillofacial region: a retrospective study of 130 cases in a southern Iranian population. Pathology research international. 2011; 2011.

22) Bouq Y, Fazili FM, Gaffar HA. A current pattern of surgically treated thyroid diseases in the Medinah region of Saudi Arabia. JK-Practitioner. 2006; 13: 9-14.

23) Khademi B, Taraghi A, Mohammadian Panah M. Anatomical and histopathological profile of head and neck neoplasms in Persian pediatric and adolescent population. Int J Pediatr Otorhinolaryngol. 2009; 73 : 1249-53. doi: 10.1016/j.ijporl.2009.05.017. PMID: 19525017.

24) Refeidi AA, Al-Shehri GY, Al-Ahmary AM, Tahtouh MI, Alsareii SA, Al-Ghamdi AG, et al. Patterns of thyroid cancer in Southwestern Saudi Arabia. Saudi Med J. 2010; 31: 1238-41. PMID: 21063655.

25) Al-Amri AM. Pattern of thyroid cancer in the eastern province of Saudi Arabia: University hospital experience. Journal of cancer therapy. 2012; 3: 187-91.

26) Blot WJ, McLaughlin JK, Winn DM, Austin DF, Greenberg RS, Preston-Martin S, et al. Smoking and drinking in relation to oral and pharyngeal cancer. Cancer Res. 1988; 48: 3282-7. PMID: 3365707.

27) Abdel Rahim BE, Mahfouz MS, Yagoub U, Solan YM, Alsanosy RM. Practice and attitude of cigarette smoking: a community-based study. PloS one. 2014; 9: e92939. doi: 10.1371/journal.pone.0092939. PMID: 24695369, PMCID: PMC3973620.

28) Al-Idrissi H. Head and neck cancer in Saudi Arabia: Retrospective analysis of 65 patients. J Int Med Res. 1990; 18: 515-9. doi: 10.1177/030006059001800610. PMID: 2292333.

29) Goins MR, Beasley MS. Pediatric Neck Masses. Oral and Maxillofacial Surgery Clinics. 2012; 24: 457-68. doi: 10.1016/j.coms.2012.05.006.

30) Dickson PV, Davidoff AM. Malignant neoplasms of the head and neck. Seminars in Pediatric Surgery. 2006; 15: 92-8.

31) Jiang H, Tian Y, Yan W, Kong Y, Wang H, Wang A, et al. The Prevalence of Thyroid Nodules and an Analysis of Related Lifestyle Factors in Beijing Communities. Int J Environ Res Public Health. 2016; 13(4): 442. doi: 10.3390/ijerph13040442. PMID: 27110805, PMCID: PMC4847104.

32) Popoveniuc G, Jonklaas J. Thyroid nodules. Med Clin North Am. 2012; 96(2): 329-49. doi: 10.1016/j.mcna.2012.02.002. PMID: 22443979, PMCID: PMC3575959.

33) Markou K, Christoforidou A, Karasmanis I, Tsiropoulos G, Triaridis S, Constantinidis I, et al. Laryngeal cancer: epidemiological data from Northern Greece and review of the literature. Hippokratia. 2013; 17: 313-8. PMID: 25031508, PMCID: PMC4097410.

34) Yun YH, Jung KW, Bae JM, Lee JS, Shin SA, Min Park S, et al. Cigarette smoking and cancer incidence risk in adult men: National Health Insurance Corporation Study. Cancer Detect Prev. 2005; 29: 15-24. doi: 10.1016/j.cdp.2004.08.006. PMID: 15734213. 